\title{
Finding big shots: small-area mapping and spatial modelling of obesity among Swiss male conscripts
}

\author{
Radoslaw Panczak ${ }^{1,2}$, Leonhard Held ${ }^{3}$, André Moser ${ }^{2}$, Philip A. Jones ${ }^{4}$, Frank J. Rühli ${ }^{1}$ and Kaspar Staub ${ }^{1 *}$
}

\begin{abstract}
Background: In Switzerland, as in other developed countries, the prevalence of overweight and obesity has increased substantially since the early 1990s. Most of the analyses so far have been based on sporadic surveys or self-reported data and did not offer potential for small-area analyses. The goal of this study was to investigate spatial variation and determinants of obesity among young Swiss men using recent conscription data.

Methods: A complete, anonymized dataset of conscription records for the 2010-2012 period were provided by Swiss Armed Forces. We used a series of Bayesian hierarchical logistic regression models to investigate the spatial pattern of obesity across 3,187 postcodes, varying them by type of random effects (spatially unstructured and structured), level of adjustment by individual (age and professional status) and area-based [urbanicity and index of socio-economic position (SEP)] characteristics.
\end{abstract}

Results: The analysed dataset consisted of 100,919 conscripts, out of which 5,892 (5.8\%) were obese. Crude obesity prevalence increased with age among conscripts of lower individual and area-based SEP and varied greatly over postcodes. Best model's estimates of adjusted odds ratios of obesity on postcode level ranged from 0.61 to 1.93 and showed a strong spatial pattern of obesity risk across the country. Odds ratios above 1 concentrated in central and north Switzerland. Smaller pockets of elevated obesity risk also emerged around cities of Geneva, Fribourg and Lausanne. Lower estimates were observed in North-East and East as well as south of the Alps. Importantly, small regional outliers were observed and patterning did not follow administrative boundaries. Similarly as with crude obesity prevalence, the best fitting model confirmed increasing risk of obesity with age and among conscripts of lower professional status. The risk decreased with higher area-based SEP and, to a lesser degree - in rural areas.

Conclusion: In Switzerland, there is a substantial spatial variation in obesity risk among young Swiss men. Small-area estimates of obesity risk derived from conscripts records contribute to its understanding and could be used to design further studies and interventions.

Keywords: Obesity, Switzerland, Conscripts, Disease mapping, Spatial hierarchical Bayesian analysis, Integrated nested Laplace approximation

\footnotetext{
*Correspondence: kaspar.staub@iem.uzh.ch

${ }^{1}$ Institute of Evolutionary Medicine, University of Zurich, Winterthurerstrasse

190, CH-8057 Zurich, Switzerland

Full list of author information is available at the end of the article
} 


\section{Background}

The prevalence of overweight and obesity (OWOB), with steady increase over three decades, has reached global pandemic proportions in the developed world [1-3], with over 1.4 billion adults classed as overweight in 2008. Within this figure, over 200 million men and nearly 300 million women were obese [4].

The negative associations of overweight and obesity with chronic disease such as diabetes type II, hypertension, cardiovascular disease and some cancers is well documented [5-8]. Currently there is some scientific debate regarding a possible protective effect of low-level overweight to lower mortality risk, yet the negative effect of obesity on morbidity and mortality is certain $[9,10]$. Quality of life for overweight or obese adolescents and adults has been linked to poorer health outcomes, low family income and a limiting effect on marriage possibilities later in life $[11,12]$.

From the early 1990s an increase in the prevalence of OWOB in many developed countries including Switzerland [13-15] has imposed a burden on the population and health care system [16]. In 2006, OWOB and its co-morbidities, and health consequences constituted $11 \%$ of total Swiss healthcare expenditure [17] including: 27,000 cases of diabetes type II, 63,000 cases of hypertension and 37,000 cases of lipid metabolism disorders. These health risks and costs could have been circumvented had OWOB remained at 1992 levels [5].

Research into OWOB in Switzerland comprises an important component for public health research and planning. However longitudinal, nationally measured data on the prevalence of OWOB in Switzerland is lacking [13]. A number of studies have provided socioeconomic and regional differences on the prevalence of OWOB but many of these were based on sporadic surveys with subsequently limiting data on regional, demographic and socio-economic representation [14, 18-24]. These studies might also be biased by sample selection/size and low participant numbers [14]. Self-reporting of stature and weight in some surveys such as the Swiss Health Survey (SHS) [25] and the Swiss Household Panel $[26,27]$ may also introduce bias.

These results indicate early signs of a slowdown in the increase of OWOB among both adults and children in recent years, implying some success in increased awareness of the condition and public health programmes targeting physical activity and healthy eating [22, 28-32]. Estimates of obesity prevalence varied widely, between 3 and $15 \%(B M I=>30 \mathrm{~kg} / \mathrm{m} 2)$, as a result of which sex, age, ethnic or socioeconomic group and which anthropometric measurement method was analysed. Variability between studies of obesity prevalence is substantial between waves of the SHS [25] and the various regional and epidemiological studies [13, 20,33-36]. Estimates of small-area regional variability of $\mathrm{OBOW}$ and their determinants presently exist only for the city of Geneva and such estimates are lacking elsewhere in Switzerland [37].

The complex pathogenesis of obesity has yet to be completely understood [38]. In addition to genetic and epigenetic factors [39], environmental factors, socioeconomic position, and behavioural choices such as daily food intake and energy expenditure have an impact on the risk of obesity [7]. Even public health policies like smoking bans might have unintended effects and provoke weight gain [40]. Moreover, the literature has featured a steady increase in the number of reports suggesting associations with small-area community or neighbourhood characteristics (e.g., access to playgrounds and to grocery stores with affordable healthful food), social-network effects, and home-environment factors (adequate housing, culture, parental model, etc.) on obesity [7, 41-44]. Understanding such area-based epidemiological features of obesity is an important step toward understanding its aetiology and prioritizing future public-health interventions.

The main goal of this study is to investigate small-area variation and determinants of obesity among young Swiss men using georeferenced conscription data from 2010-2012 in order to better understand spatial and socio-economic pattering of obesity in Switzerland and to assist planning interventions on a small scale.

\section{Methods}

\section{Conscript data in public health research}

Nations in Europe which continue to conscript their male citizens into military infrastructure include Finland, Norway, Denmark, Austria and Switzerland. Conscription is mandatory for all young males and entails a medical examination with recording of standardised anthropometric measurements. The process is repeated annually and provides valuable anthropometric data on young men of a directive age [45]. Though these conscription systems are not outlined for collection of such data per se, they have successfully served as a basis for epidemiological studies in Switzerland [29, 46, 47], Sweden [48, 49], Austria [50-52], and Germany [53]. Though limited to young males, the prevalence of conscripts' OBOW remains a valuable research source because obesity in young adulthood increases the likelihood of being obese later in life, and the morbidity and mortality risks of men increase with advancing age $[6,54]$.

\section{Swiss conscription process}

First instituted in 1875, the mandatory recruitment for the Swiss Armed Forces was renewed and expanded in 2004. Specifically, all young Swiss men are summoned for conscription during the year in which they get 19 but may request conscription before or after this year. 
Mandatory assessments collect data referencing anthropometric status (measured stature and body weight, rounded to integers), socioeconomic status (occupation) and place of domicile, and are required even for those who may subsequently be granted a deferral or an exemption from service. Six conscription centres (Lausanne, Sumiswald, Windisch, Rüti, Mels and Monte Ceneri) conduct professional, medically supervised assessments using uniform quality standards for technical facilities and work processes (Bundesgesetz über die Armee und die Militärverwaltung, Militärgesetz MG, 510.10, Art. 2; MG Art. 9, Verordnung über die Rekrutierung VREK, 511.11, Art. 3 and Art. 9). Measurements are continuously entered in the medical-information database of the Swiss Armed Forces (MEDISA) and are accessible to Army personnel. The resulting data reflect over $90 \%$ of the yearly male birth cohorts, allowing analyses of variation across temporal, regional and socioeconomic strata $[29,55]$.

\section{Data sources}

Anonymised individual conscription entries covering the time period of 1 January 2010 - 31 December 2012 were provided to the study authors under contractual agreement by the Swiss Armed Forces (Logistikbasis der Armee, LBA San). Retained information included date of birth, date of conscription, height and weight as measured by the army personnel, current occupation (free-text input), postcode of place of domicile and conscription status (first and regular appearance or re-evaluation). The Federal Directorate of Cadastral Surveying provided a spatial dataset of postcode boundaries (Release 7 - 1. May 2013), a version of the data with postal geography as of 31 March 2013.

\section{Data availability and ethics statement}

In accordance with Swiss law (Bundesgesetz über die militärischen Informationssysteme MIG, BG 510.91, Art. 2, 9, 24-29) the Swiss Armed Forces have authority to provide anonymized data for academic research; where analyses are based on such anonymized and nonclinical government data, ethical approval is not required (Swiss data privacy act, SR 235.1; 19.6.1992 and Federal Act on Research involving Human Beings HRA, 810.30; 1.1.2014). Thus upon approval of the project protocol by Logistikbasis der Armee - LBA San [56], a signed bilateral data contract was enacted between the study authors and the Swiss Armed Forces. Names, identification numbers and residential addresses were removed prior to data delivery. As conscription in Switzerland is mandatory and the body measurements analysed in this publication fit the profile of nonclinical government data, informed consent is not required.

\section{Study population}

The study included male conscripts between 18 and 22 years of age presenting for the first regular assessment in one of the recruitment centres. Individuals with a missing or incorrect postcode or insufficient information regarding occupational status were excluded as well as conscripts with implausible stature and weight values $(130 \mathrm{~cm}>$ height $>220 \mathrm{~cm} ; 30>$ weight $>200 \mathrm{~kg})$. Apart from one young man with a recorded weight of $500 \mathrm{~kg}$, none of the individuals were above $200 \mathrm{~kg}$. No unfeasible BMI values were found.

\section{Spatial resolution}

We used postcode of place of residence at the time of conscription as the spatial resolution for the study. Because Swiss postcode boundaries change over time (due to creation or deletion, merges, splits, etc.) we standardized postcodes of places of residence from various years to the state on the $31^{\text {st }}$ of March 2013 in order to match them to the available spatial dataset of postcode boundaries.

\section{Representativeness}

A precise assessment of the representativeness of the conscript population in comparison to the total population of young Swiss males is at present non-existent. However, an earlier study using similar sources [29] estimated data to be of high coverage ( $>90 \%$ ), despite using narrower age range of study participants (18.5-20.5 years of age).

\section{Variables}

Obesity was defined using World Health Organization $(\mathrm{WHO})$ classification as Body Mass Index $(\mathrm{BMI}=$ weight $[\mathrm{kg}] /$ height $\left.[\mathrm{m}]^{2}\right) \geq 30 \mathrm{~kg} / \mathrm{m}^{2}[57,58]$. Age at conscription was calculated based on the dates of birth and conscription, and then categorised into four one-year intervals from [18-19) to [21-22) years of age. For occupational status, a separate category for those in education was created ('Pupils') and other occupations were categorized as follows. Using the International Standard Classification of Occupations (ISCO-08) (as specified by the International Labour Organization [59]), the freetext entry for current occupation was converted, then aggregated into three major, hierarchical groups [60]: 'Low' (ISCO major groups 7-9), 'Medium' (ISCO major groups 3-6) and 'High' (ISCO major groups 1-2) [29].

Postcodes were classified into three categories ('Urban', 'Peri-urban' and 'Rural') according to the degree of urbanization of the community in which they were located using classification of the Swiss Federal Statistical Office. Finally, median value of the Swiss neighbourhood index of socioeconomic position (Swiss-SEP; [61]) was calculated for all postcodes and matched to 
the individual entries. Median postcode Swiss-SEP was then categorized into quintiles, in order of increasing socioeconomic status [29].

\section{Statistical analyses}

We calculated percentages of obese conscripts across 1year age bands, categorical variables (professional status and urbanicity) and quintiles of Swiss-SEP. Obesity was the binary outcome in all regression models. We used Bayesian hierarchical logistic regression models to investigate the spatial pattern of obesity across postcodes [62-64]. Since our main hypothesis was that obesity geographically varies across postcodes, we developed a series of models that differed in use of the unstructured and spatially structured random components and the level of adjustment in order to investigate the influence of the spatial dependence between postcodes: 1) a spatially unstructured component (i.e. area-independent effect) and no covariates (Model 1); 2) Model 1 with adjustment covariates (Model 2); 3) a spatially unstructured component and a spatially structured component (i.e. spatial effect depending on neighbouring postcodes; modelled using intrinsic conditionally autoregressive (iCAR) model) [65] without covariates (Model 3); 4) Model 3 with adjustment covariates (Model 4). CAR models in Bayesian framework allowed us to incorporate measures of connectivity between regions allowed to smooth the relative risk estimate in given area towards the mean risk in the neighbouring regions. Such approach of 'borrowing information' from adjacent areas allows obtaining more reliable risk estimates in comparison to using the crude rates, particularly for regions with small count of events [66]. Adjusted models used two individual (age and professional status) and two area-based characteristics (urbanicity and Swiss-SEP). In additional models we compared use of 1 year age bands and quintiles of Swiss-SEP with continuous variables and tested for potential influence of the recruitment centre. Similarly to other studies [63, 67-69], unstructured and structured random effects were modelled as independent zero-mean Gaussian random variables with vague gamma $(1,0.01)$ and gamma $(1,0.001)$ priors on the corresponding precision parameters. For the fixed effects we used zero-mean Gaussian priors with variance 1000. We used the deviance information criterion (DIC) for comparing model fits [70].

We used 'queen' contiguity-based spatial weights where all postcodes sharing any length of the border (including corners) to conceptualize neighbourhood relationships [71]. All analyses were done in $\mathrm{R}$ 3.1.1 [72] using the R- INLA library [[69], www.r-inla.org]. INLA implements the integrated nested Laplace approximation approach for latent Gaussian models [69].

Results are presented as combined proportional symbol [73] and choropleth maps. Squares placed at the median population centre of each postcode represent its estimated odds ratios (ORs) with hue, their size scaled according to the square root of the total number of conscripts from 2010-12 period. Population, and proportionally with it - conscripts, is very unevenly distributed in Switzerland, with large areas of the Alps having few or no residents. Proportional symbol mapping approach assured that postcodes with large area and sparse conscript numbers are less prominent on the map. Bayesian posterior probabilities of obesity for each postcode are shown in the same maps as an underlying layer, in shades of green. Darker hues were chosen for postcodes with higher probability. Both colour schemes were derived from ColorBrewer.org tool [[74], colorbrewer.org]. Diverging colour scheme for ORs was modified by skipping middle, light hues to improve map readability.

\section{Results}

\section{Study population and crude obesity prevalence across} postcodes

The initial sample consisted of 123,357 conscripts. We excluded $802(0.7 \%)$ women, 4,549 (3.7 \%) conscripts appearing for reassessment, five $(<0.1 \%)$ with implausible values of height and weight, one $(<0.1 \%)$ with missing information of postcode of place of residence, 16 $(<0.1 \%)$ below 18 and 6,690 (5.4\%) above 22 years old and 10,375 (8.4\%) with missing or imprecise occupation. The final sample consisted of 100,919 conscripts, out of which 5,892 (5.8 \%) were obese.

On 31.03.2012 there were 3,187 postcodes in Switzerland. The mean number of conscripts in postcode was 31.7 (standard deviation: 44.9) and ranged from zero (in 102 postcodes) to 505 (one postcode). Due to small population sizes there was a great variation in crude percentage of obese conscripts across postcodes ( rang from 0.0 to $100.0 \%)$.

\section{Obesity prevalence across socioeconomic characteristics}

Obesity prevalence varied across sociodemographic characteristics of conscripts and by the types of areas they came from. Figure 1 shows crude percentages and their $95 \%$ confidence intervals (CI) of obesity prevalence. There was a clear gradient of increasing percentage of obese individuals across 1 year age groups: There were $7.0 \%(6.4-7.5 \% 95 \% \mathrm{CI})$ obese conscripts among 21 year old conscripts as opposed to $4.8 \%(4.6-5.1 \%$ $95 \% \mathrm{CI}$ ) among 18 year olds. The opposite gradient of similar strength was observed across strata of professional status with $4.6 \%(4.3-4.9 \% 95 \% \mathrm{CI})$ obese conscripts in the 'High' group as opposed to the 'Low' $6.8 \%(6.6-7.1 \% 95 \%$ CI). A similar inverse gradient existed between $1^{\text {st }}$ (highest SEP) and $5^{\text {th }}$ (lowest SEP) quintiles of neighbourhood SEP index. Differences 


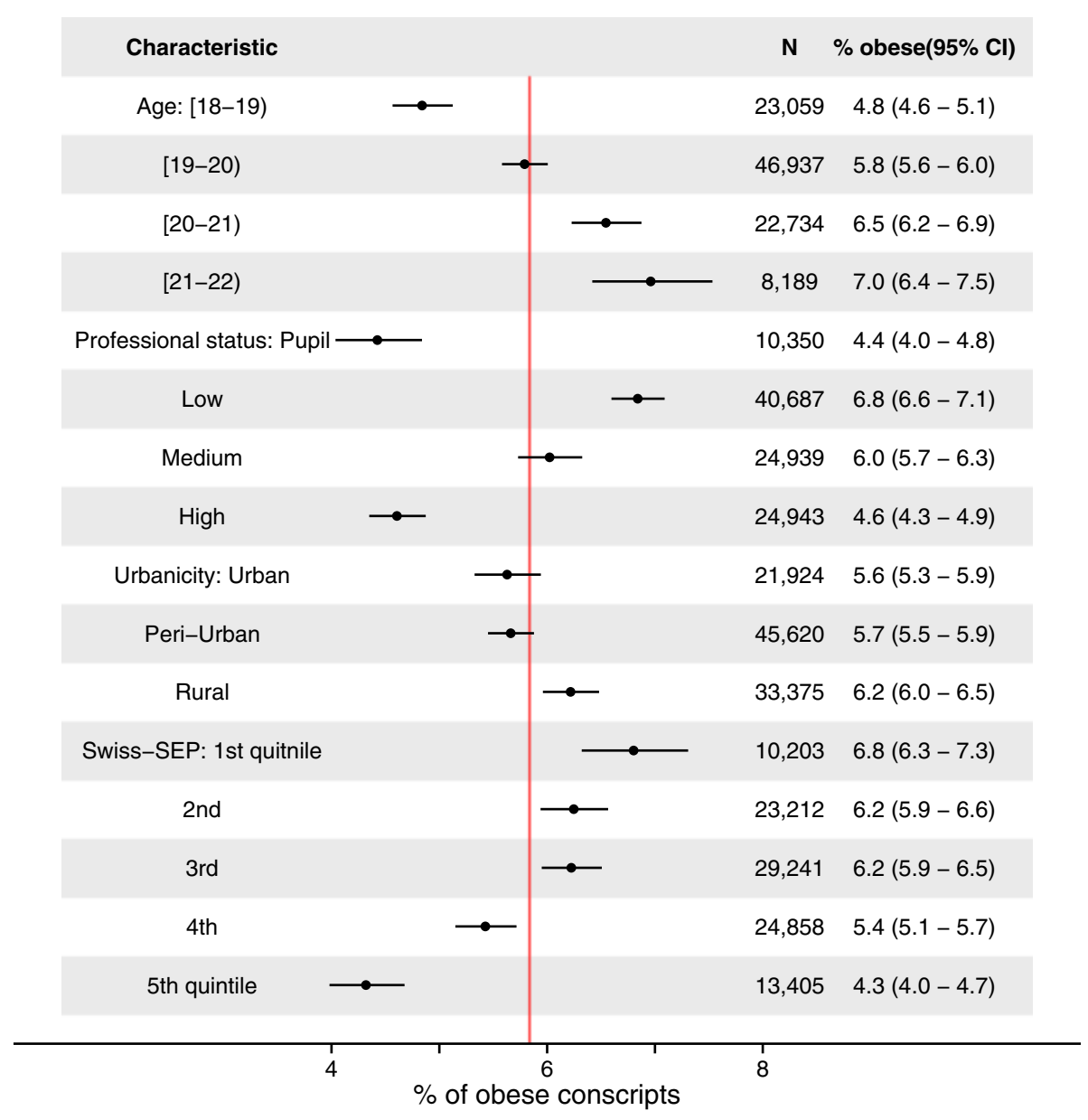

Fig. 1 Crude prevalence of obesity across age and socioeconomic characteristics of conscripts, Switzerland 2010-12. Vertical red solid line indicates $5.8 \%$ overall proportion of obesity

between conscripts coming from communities of different urbanicity levels were less pronounced.

\section{Obesity prevalence across postcodes}

Figure 2 shows postcode level estimates of ORs of obesity among conscripts from unadjusted (Model 1) and Fig. 3 shows the adjusted model with spatially unstructured component (Model 2). Adjustment for individual and area level covariates did not cause major changes in the spatial patterning of estimates. In both models there was a heterogeneity of ORs between postcodes. Some large scale spatial patterns emerged from the maps indicating SW-NE belt of elevated OR s in Central and North Switzerland (stretching mainly throughout postcodes located in the cantons of Basel Stadt and Basel Land, Solothurn, Aargau and northern parts of Bern and Zurich) [75]. Postcodes with ORs below 1.0 were concentrated in NE Switzerland (the cantons of Zurich and Thurgau) and scattered through the rest of the country. Posterior probability estimates were relatively low throughout whole country. DIC indicated Model 2 as having better fit compared to Model 1 (Table 1).
Figure 4 shows postcode level estimates of ORs of obesity from unadjusted (Model 3) and Fig. 5, the adjusted model with spatially unstructured and structured components (Model 4). Similarly as in two previous models, adjustment for individual and area level covariates did not cause major changes in the spatial patterning of estimates. The heterogeneity of ORs increased with postcode-level estimates ranging from 0.61 to 1.93 , further accentuating strong spatial structure of the results. There was a clear concentration of ORs above 1 in central and north Switzerland. Additional, smaller pockets of postcodes with higher ORs emerged around cities of Geneva, Fribourg and Lausanne. Lower estimates of ORs were observed in the North-East (cantons Thurgau, Schaffhausen, St. Gallen, Appenzell Ausserrhoden, Appenzell Innerrhoden) and East (canton Grisons) Switzerland as well as south of the Alps (in the cantons of Valais and Ticino). In contrast to the results from models without structured components, posterior probability estimates were higher in North, Central and Western part of the country. 


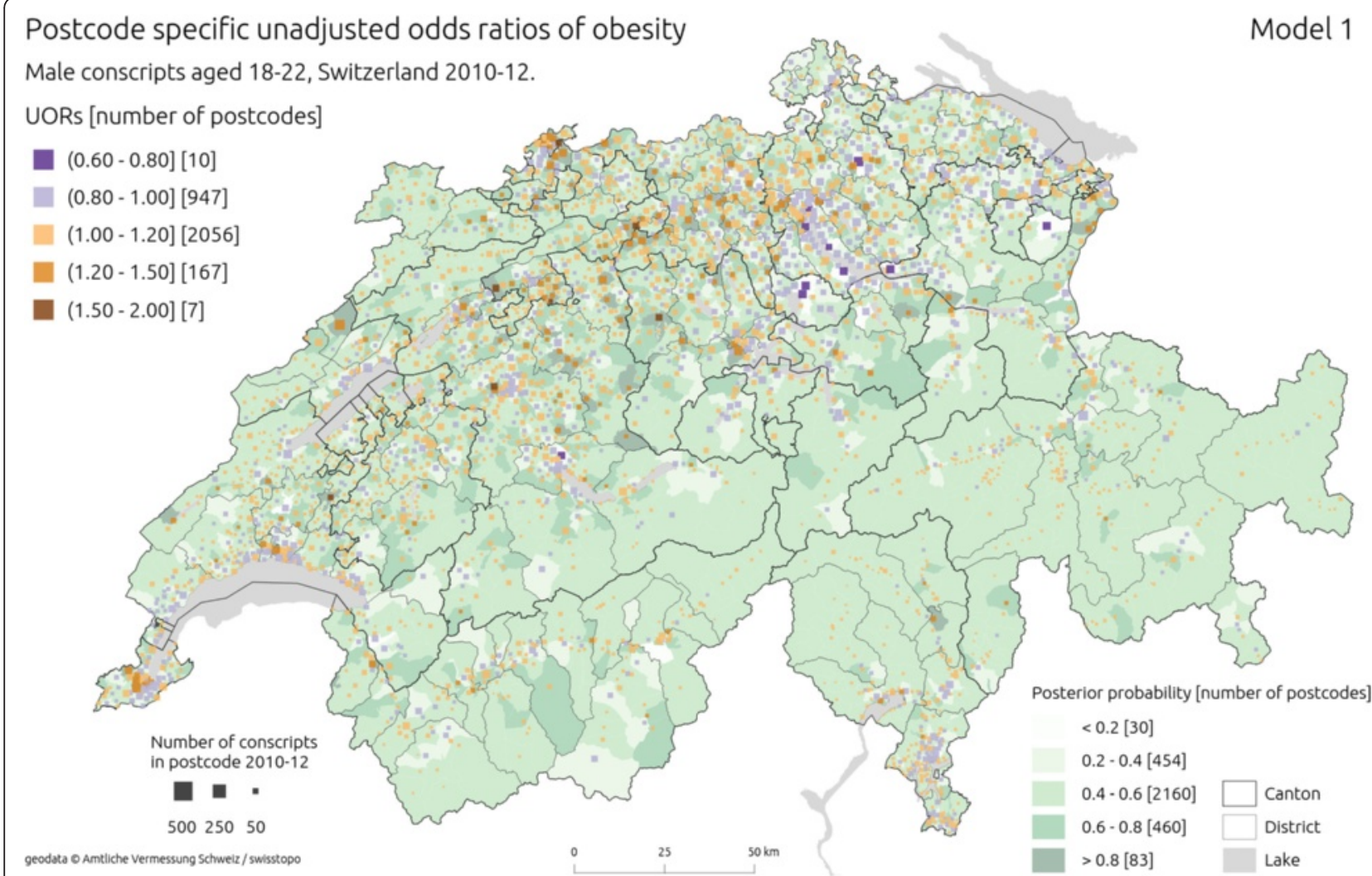

Fig. 2 Estimated unadjusted odds ratios (UORs) of obesity from Model 1 ( using spatially unstructured effects). The squares are placed in the median population centres of the postcodes from which conscripts came from; the hue of the square is shaded according to the estimated OR in a given postcode, and its size is proportional to the square root of the number of conscripts from 2010-12 period. Background choropleth map (green hues) represents posterior probability of an increased risk of obesity. Sources of geodata: Amtliche Vermessung Schweiz/swisstopo

\section{Comparison of the models}

DIC indicated Model 4 as having best fit to the data (Table 1). Adding the spatial component helped to explain the variation in the data (more total variance), whereas adding covariates reduced the total variance (0.016 difference between Model 1 and 2, and 0.012 between 3 and 4), but not really the spatial variance (0.001 difference between Model 3 and 4) (Table 1). Lastly, there was a noted change in the shape of distribution of postcode specific estimates of ORs between models, particularly when the spatial effects were included (Additional file 1: Figure S1). Whereas the median of the estimates was in the 0.99-1.02 range for all four models, the IQR range increased with complexity of the models and ranged 0.99-1.08, 0.99-1.06, 0.89-1.18, 0.85-1.19 for models 1 to 4 respectively.

\section{Association with individual and area-based characteristics}

Table 1 shows the association of the covariates (fixed effects) from adjusted models 2 and 4. There was little change between the estimates after adding the spatially structured effects. The results of the best fitting model 4 confirmed most of the effects seen in crude prevalence estimates (Fig. 1). There was a significant positive effect of age [adjusted OR 1.18 per 1 year of age; $1.14-1.22$ $95 \%$ credible interval $(\mathrm{CI})]$ and negative effect of individual's professional status (adjusted OR 0.70, 0.63$0.7995 \%$ CI comparing 'Low' to 'Medium'). The association with Swiss-SEP index (reported as per 10 units increase; the original Swiss-SEP ranges from 0 to 100) was almost equal in strength (adjusted OR $0.79,0.75-0.8495 \% \mathrm{CI})$.

Lastly, there was little effect of urbanicity of place of residence with conscripts from rural communities having slightly lower odds of being obese. In the additional model we included random effect of the conscription centre to check for any potential differences between them, but failed to find any association (results not shown).

\section{Discussion}

The aim of this study was to describe small-area variation and socioeconomic determinants of obesity among young men in Switzerland using recent waves of conscription data. Using records from 2010-12 period, we found a strong spatial structure of risk of obesity across 


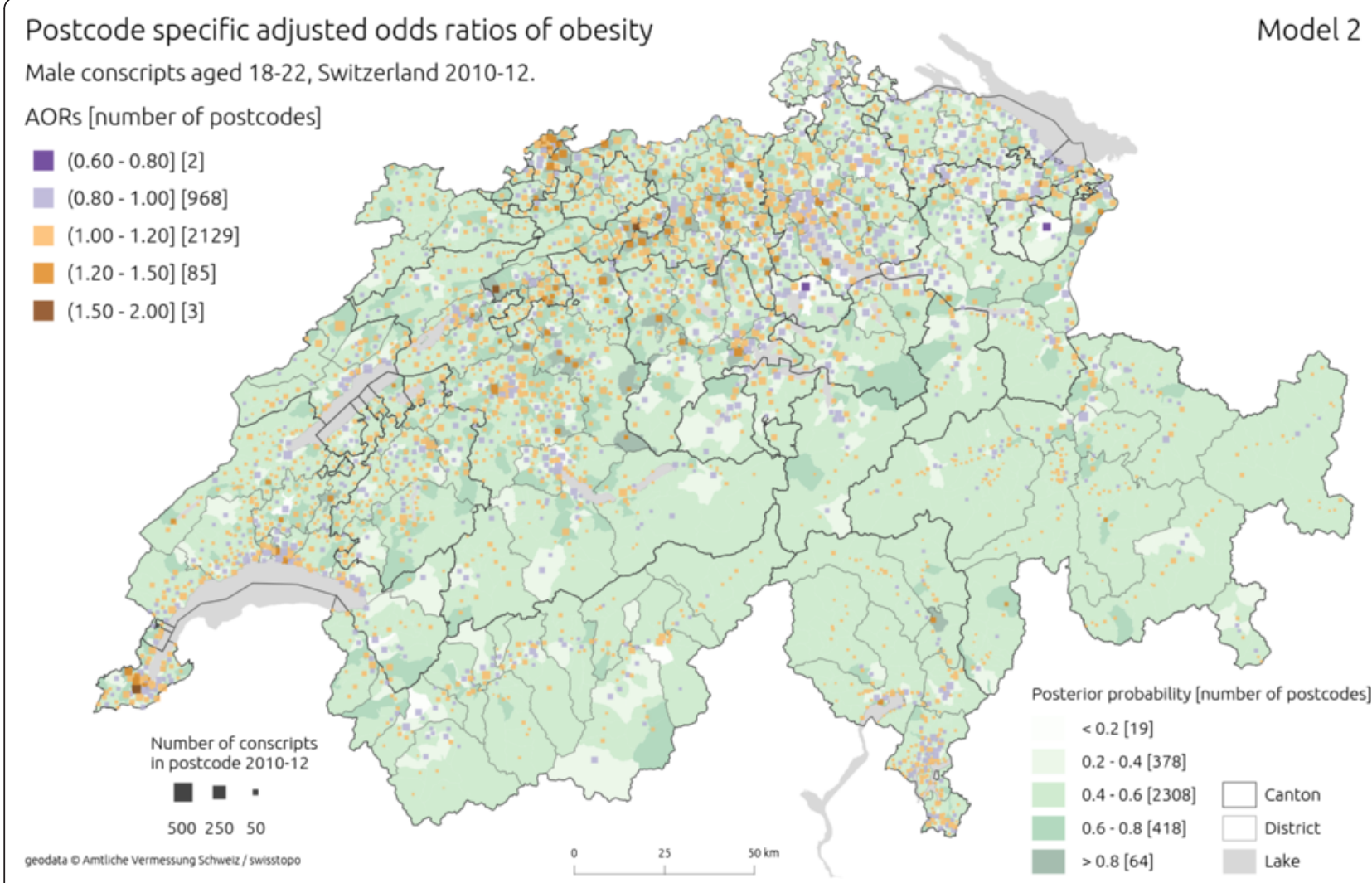

Fig. 3 Estimated adjusted odds ratios (AORs) of obesity from Model 2 ( using spatially unstructured effects). The squares are placed in the median population centres of the postcodes from which conscripts came from; the hue of the square is shaded according to the estimated OR in a given postcode, and its size is proportional to the square root of the number of conscripts from 2010-12 period. Background choropleth map (green hues) represents posterior probability of an increased risk of obesity. Sources of geodata: Amtliche Vermessung Schweiz/swisstopo

Table 1 Odds ratios across the covariates (fixed effects) together with their $95 \%$ credible interval and model parameters of four hierarchical logistic regression models. See text for the detailed description of differences between models

\begin{tabular}{|c|c|c|c|c|c|}
\hline Variable & Category & Model 1 & Model 2 & Model 3 & Model 4 \\
\hline Age & (per 1 year) & - & $1.17(1.13-1.21)$ & - & $1.18(1.14-1.22)$ \\
\hline \multirow[t]{4}{*}{ Professional status } & Pupil & - & $0.75(0.67-0.84)$ & - & $0.70(0.63-0.79)$ \\
\hline & Low & - & $1.10(1.03-1.17)$ & - & $1.10(1.03-1.17)$ \\
\hline & Medium & - & 1.00 (ref.) & - & 1.00 (ref.) \\
\hline & High & - & $0.73(0.68-0.79)$ & - & $0.74(0.68-0.80)$ \\
\hline Swiss-SEP & (per 10 units) & - & $0.83(0.80-0.87)$ & - & $0.79(0.75-0.84)$ \\
\hline \multirow[t]{3}{*}{ Urbanicity } & Urban & - & $0.97(0.89-1.06)$ & - & $1.01(0.93-1.09)$ \\
\hline & Peri-urban & - & 1.00 (ref.) & - & 1.00 (ref.) \\
\hline & Rural & - & $0.91(0.84-0.98)$ & - & $0.90(0.83-0.98)$ \\
\hline \multirow[t]{2}{*}{ Variance of random effects } & Total & 0.073 & 0.057 & 0.086 & 0.074 \\
\hline & Spatial component & - & - & 0.065 & 0.065 \\
\hline DIC & & $44,812.27$ & $44,511.27$ & $44,696.30$ & $44,358.98$ \\
\hline pD & & 308.59 & 260.16 & 214.66 & 150.35 \\
\hline
\end{tabular}




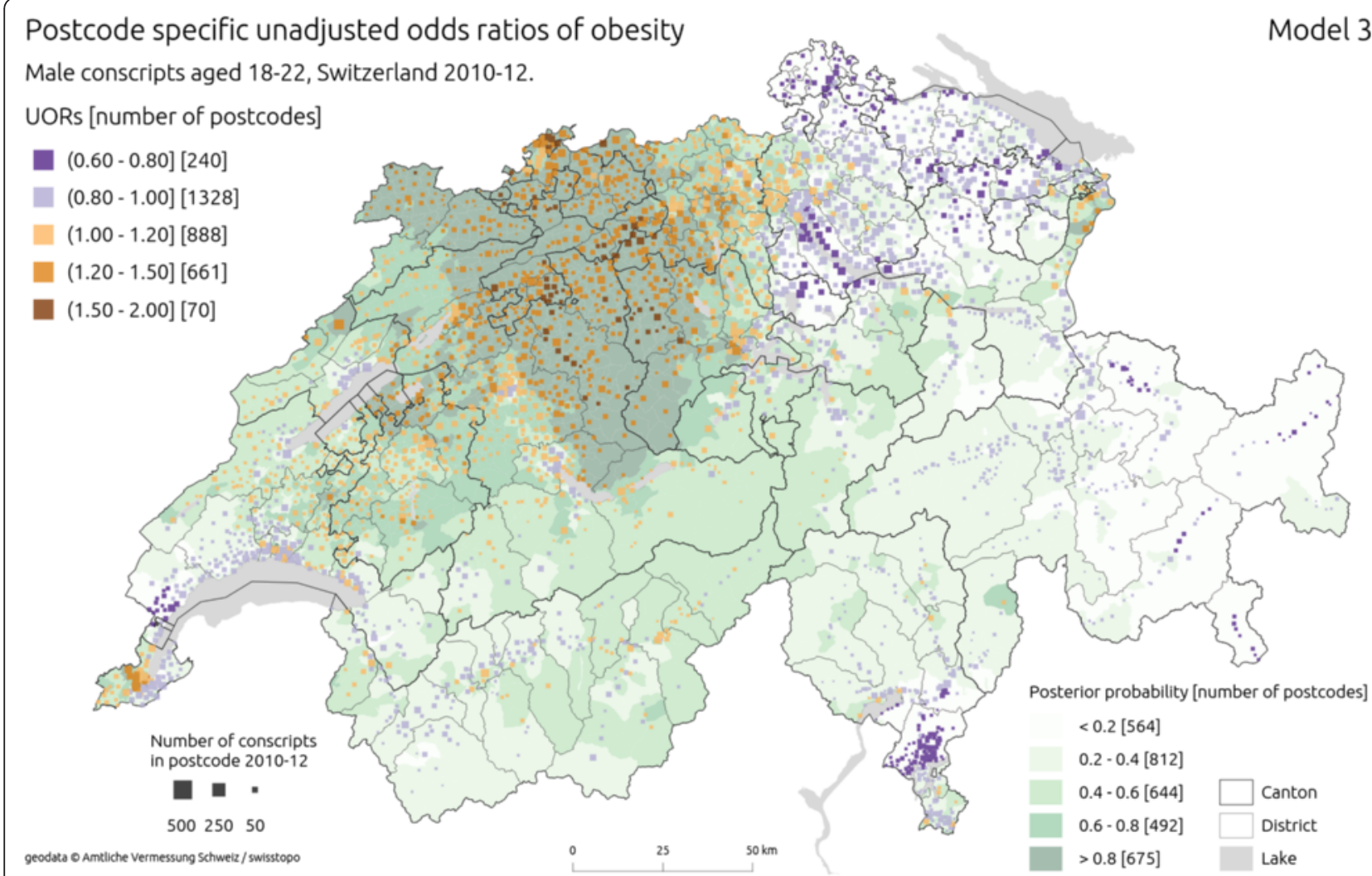

Fig. 4 Estimated unadjusted odds ratios (UORs) of obesity from Model 3 ( using spatially unstructured and structured effects). The squares are placed in the median population centres of the postcodes from which conscripts came from; the hue of the square is shaded according to the estimated OR in a given postcode, and its size is proportional to the square root of the number of conscripts from 2010-12 period. Background choropleth map (green hues) represents posterior probability of an increased risk of obesity. Sources of geodata: Amtliche Vermessung Schweiz / swisstopo

postcodes. A clear concentration of high and low values of ORs emerged, but also small, regional outliers were observed. Importantly, spatial patterning did not always follow administrative boundaries of large administrative units [cantons and districts (Bezirke)] and would be missed or distorted if they were used for the analysis. Lastly, results indicated age and socioeconomic gradients in obesity risk, with smaller influence of urbanicity.

The main strength of this study is its reliance on representative, large and objectively measured georeferenced data. The sample size allowed high-resolution analysis on the level of individual postcodes. Such findings could contribute to the understanding of the small-area obesity risk that not necessarily follows administrative boundaries (cantons) and thus help to design further studies and target interventions. The study however has several limitations and any generalisation of the results should take them into account. First of all, this study focusses on young, Swiss men only and the associations cannot be directly applied to women, other age groups and non-Swiss individuals. BMI driven obesity indicator was the only body shape measure available for this study. Because BMI cannot accurately differentiate between weight associated with fat and lean muscle mass, it not an ideal measure of body composition [76-78]. This can occasionally, lead to misclassification of physically very fit individuals, especially in the overweight BMI category 25.0$29.9 \mathrm{~kg} / \mathrm{m}^{2}$. Nevertheless, BMI is strongly correlated with the body fat percentage particularly regarding the categories of obesity (BMI $\geq 30 \mathrm{~kg} / \mathrm{m}^{2}$ ) and because of its ease of use and availability it is the most commonly utilised measure for large-scale investigations and convenient assessment in clinical practice $[7,79,80]$. By relying on standard definition of obesity, we avoided problem of ad hoc and post hoc category selection and assured comparability with other studies [81]. Additionally, profession is a limited indicator for socioeconomic background of young individuals, because a significant number of conscripts were still in education [47, 82]. We attempted to mitigate that limitation by using area-based SEP index, which at the moment is the only such indicator available in Switzerland [61]. Further, conscription data do not provide information about the migration background of the young men. Lastly, residential postcode of the conscripts was the only geographical variable available in the dataset. Swiss postcodes are still arbitrary geographical units, which were 


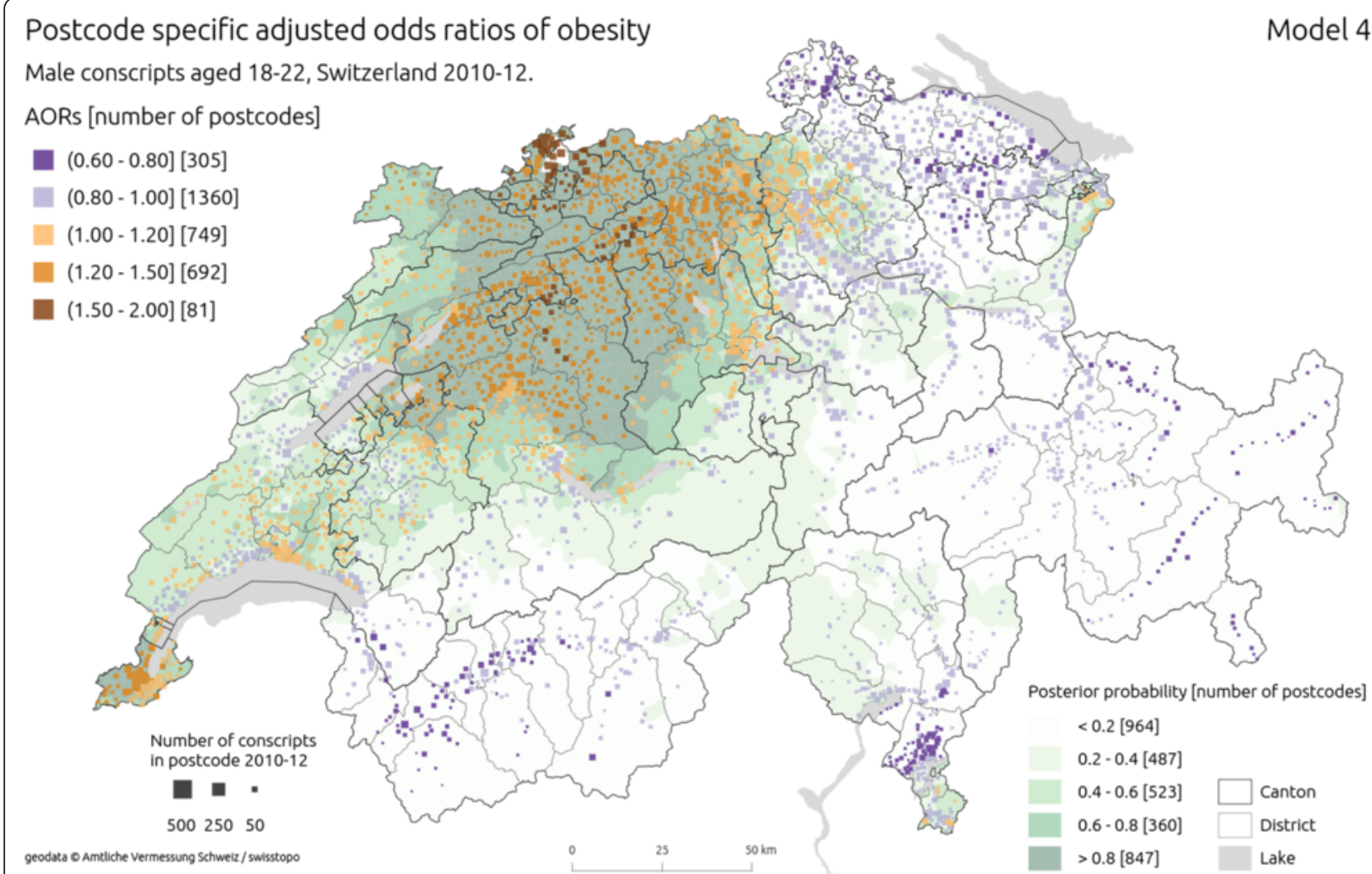

Fig. 5 Estimated adjusted odds ratios (AORs) of obesity from Model 4 (AOR), (using spatially unstructured and structured effects). The squares are placed in the median population centres of the postcodes from which conscripts came from; the hue of the square is shaded according to the estimated OR in a given postcode, and its size is proportional to the square root of the number of conscripts from 2010-12 period. Background choropleth map (green hues) represents posterior probability of an increased risk of obesity. Sources of geodata: Amtliche Vermessung Schweiz/swisstopo

not designed for public health investigations. However, they offer a high level of resolution for national scale research whilst still maintaining privacy of individuals. Additionally, they remain in use in official health statistics as a basis for formulation of MedStat regions [83] and can therefore in the future be linked to other Swiss health related data such as the Swiss database of hospital discharges (Medizinische Statistik der Krankenhäuser).

Obesity prevalence among Swiss conscripts 2010-2012 (5.8 \%) was slightly higher compared to the prevalence (3.6\%) detected among the small group of 165 men aged 15 to 29 years examined for the 2011 Swiss salt survey (however, $6.1 \%$ of the same young men showed a substantially increased waist circumference $(>102 \mathrm{~cm})$ $[34,35]$. Obesity prevalence $\left(B M I \geq 30 \mathrm{~kg} / \mathrm{m}^{2}\right)$ of 1,503 young men aged 15-24 participating in SHS in 2012 was $3.2 \%$ and thus also lower compared with the conscripts, possibly due to the fact that SHS data were self-reported [25]. The levels of obesity (BMI $\left.\geq 30 \mathrm{~kg} / \mathrm{m}^{2}\right)$ were slightly lower in Switzerland (5.8 \%) compared to other European countries with mandatory conscription in 2010/2011 (Germany $8.5 \%$, Denmark $8.7 \%$, Austria 8.4 \%) [52, 84]. In Austria, obesity prevalence among the conscripts varies between $6.9 \%$ and $9.6 \%$ between the three major regions of the country [52]. To our knowledge, this current study is the first in Switzerland, and generally the first based on conscription data, to analyse small-area variation in obesity prevalence on national level. For Switzerland, the highest resolution of regional analyses to date were cantons, major regions or districts $[14,55]$. Lastly, the study confirms results of earlier studies on Switzerland and other countries showing higher BMI among individuals of lower socio-economic position [18-20].

Future studies could offer even higher spatial resolution of the analyses and use the main city of residence or even full residential addresses geocoded to point data instead of the data aggregated to postcodes. Furthermore, more conscription years could be included, which would allow use of spatio-temporal models [63, 85]. Future studies could explore non-linear methods of modelling continuous covariates and alternative, non-contiguity-based spatial weights such as road network connectivity as suggested by recent studies on mortality and life expectancy in Switzerland [61, 86]. They should also aim at capturing highest possible level of spatial reference of place of residence of the study participants in order to further investigate complex spatial 
patterns of OWOB. Lastly, and more generally - because of the on-going nature of conscription, the dataset together with spatial models could facilitate development of the monitoring system.

\section{Conclusion}

The present study contributes to the understanding of spatial and socio-economic pattering of obesity in Switzerland. The results indicate large discrepancies in the risk of obesity among Swiss conscripts and could assist planning interventions on a small scale, irrespectively of administrative boundaries. Research on obesity and other branches of public health in Switzerland might benefit from leaving the usage of the common administrative boundaries behind.

\section{Additional file}

Additional file 1: Figure S1. Distribution of postcode level estimated odds ratios (ORs) of obesity from unadjusted (top panel) and adjusted (bottom panel) models with various use of random effects. (PDF $18 \mathrm{~kb}$ )

\section{Competing interests}

The authors declare that they have no competing interests.

\section{Authors' contributions}

Conceived and designed the experiments: RP AM LH FJR KS. Analysed the data: RP LH AM. Wrote first draft: RP. Wrote the paper: all authors. Designed cartography: RP. Obtained funding: KS FJR. Obtained data: KS FJR. All authors read and approved the final manuscript.

\section{Acknowledgements}

Swiss Federal Office of Public Health and Mäxi Foundation (for funding); Andreas Stettbacher, Chief Medical Surgeon of the Swiss Armed Forces (for providing the individual conscription data); Franz Frey, Tiziano Angelelli, Adrian Trapp, Matthias Egger, Dominik Schori, Karen Hofmann, Thomas Abel, David Faeh, Marcel Zwahlen, Matthias Bopp, Nadine Stoffel-Kurt, Andrea Poffet, Kathrin Favero, Thomas Wyss, Hans-Peter Stamm, Marek Brabec, Garyfallos Konstantinoudis, Cecilia Collins and Marta Blangiardo (for helpful comments).

\section{Author details}

${ }^{1}$ Institute of Evolutionary Medicine, University of Zurich, Winterthurerstrasse 190, CH-8057 Zurich, Switzerland. ${ }^{2}$ Institute of Social and Preventive Medicine, University of Bern, Finkenhubelweg 11, CH-3012 Bern, Switzerland. ${ }^{3}$ Epidemiology, Biostatistics and Prevention Institute, University of Zurich, Hirschengraben 84, CH-8001 Zurich, Switzerland. ${ }^{4}$ Department of Geography, Swansea University, Wallace Building, Singleton Park, Swansea SA2 8PP, UK.

Received: 18 July 2015 Accepted: 10 February 2016

Published online: 18 February 2016

\section{References}

1. Finucane MM, Stevens GA, Cowan MJ, Danaei G, Lin JK, Paciorek CJ, et al. National, regional, and global trends in body-mass index since 1980: systematic analysis of health examination surveys and epidemiological studies with 960 country-years and 9.1 million participants. Lancet. 2011:377:557-67.

2. James PT, Leach R, Kalamara E, Shayeghi M. The worldwide obesity epidemic Obes Res. 2001;9 Suppl 4:228-33.

3. Ng M, Fleming T, Robinson M, Thomson B, Graetz N, Margono C, et al. Global, regional, and national prevalence of overweight and obesity in children and adults during 1980-2013: a systematic analysis for the Global Burden of Disease Study 2013. Lancet. 2014;6736:1-16.

4. Obesity and overweight. Updated fact sheet No 311 [http://www.who.int mediacentre/factsheets/fs311/en/]
5. Davin C, Vollenweider P, Waeber G, Paccaud F, Marques-Vidal P. Cardiovascular risk factors attributable to obesity and overweight in Switzerland. Nutr Metab Cardiovasc Dis. 2012;22:952-8.

6. Engeland A, Bjorge T, Sogaard AJ, Tverdal A. Body mass index in adolescence in relation to total mortality: 32-year follow-up of 227,000 Norwegian boys and girls. Am J Epidemiol. 2003;157:517-23.

7. Kit BK, Ogden CL, Flegal KM. Epidemiology of obesity. In: Ahrens W, Pigeot I, editors. Handbook of epidemiology. 2nd ed. New York: Springer Science+Business Media; 2014. p. 2229-62.

8. De Mutsert R, Sun Q, Willett WC, Hu FB, van Dam RM. Overweight in early adulthood, adult weight change, and risk of type 2 diabetes, cardiovascular diseases, and certain cancers in men: a cohort study. Am J Epidemiol. 2014;179: 1353-65.

9. Flegal K, Bk K, Orpana H, Graubard B. Association of all-cause mortality with overweight and obesity using standard body mass index categories: a systematic review and meta-analysis. JAMA. 2013;309:71-82.

10. Tobias DK, Hu FB. Does being overweight really reduce mortality? Obesity. 2013;21:1746-9.

11. Gortmaker SL, Must A, Perrin JM, Sobol AM, Dietz WH. Social and economic consequences of overweight in adolescence and young adulthood. N Engl Med. 1993;329:1008-12.

12. Dey M, Gmel G, Mohler-Kuo M. Body mass index and health-related quality of life among young Swiss men. BMC Public Health. 2013;13:1028.

13. Faeh D, Matzke A. Ernährung und Gesundheit. In: Federal Office of Public Health, editor. Sechster Schweizerischer Ernährungsbericht. Bern: Merkur Druck AG; 2012. p. 128-208.

14. Faeh D: Epidemiologie von Übergewicht und Adipositas bei Zürcher Erwachsenen. Serie Gesundheit, Gesundheitsförderung und Gesundheitswesen im Kanton Zürich: Institut für Sozial- und Präventivmedizin der Universität Zürich; 2013;18(Mai):1-7.

15. Morabia A, Constanza M, Costanza MC. The obesity epidemic as harbinge of a metabolic disorder epidemic: trends in overweight, hypercholesterolemia, and diabetes treatment in Geneva, Switzerland, 1993-2003. Am J Public Health. 2005;95:632-5.

16. Specchia ML, Veneziano MA, Cadeddu C, Ferriero AM, Mancuso A, lanuale C, et al. Economic impact of adult obesity on health systems: a systematic review. Eur J Public Health. 2014;25(2):255-62.

17. Schneider H, Venetz W, Gallani-Berado C. Overweight and obesity in Switzerland: overweight and obesity trends in children. Basel: Federal Office of Public Health; 2009

18. Faeh D, Braun J, Bopp M. Prevalence of obesity in Switzerland 1992-2007: the impact of education, income and occupational class. Obes Rev. 2010;12:151-66.

19. Galobardes B, Costanza MC, Bernstein MS, Delhumeau C, Morabia A. Trends in risk factors for lifestyle-related diseases by socioeconomic position in Geneva, Switzerland, 1993-2000: health inequalities persist. Am J Public Health. 2003;93: $1302-9$

20. Marques-Vidal P, Bovet P, Paccaud F, Chiolero A. Changes of overweight and obesity in the adult Swiss population according to educational level, from 1992 to 2007. BMC Public Health. 2010;10:87.

21. Tschumper A, Nägele C, Alsaker FD. Gender, type of education, family background and overweight in adolescents. Int J Pediatr Obes. 2006;1:153-60.

22. Faeh D, Bopp M. Increase in the prevalence of obesity in Switzerland 1982 2007: birth cohort analysis puts recent slowdown into perspective. Obesity. 2010;18:644-6.

23. Wolff H, Delhumeau C, Beer-Borst S, Golay A, Costanza MC, Morabia A Converging prevalences of obesity across educational groups in Switzerland. Obesity. 2006:14:2080-8.

24. Zellweger U, Bopp M, Holzer BM, Djalali S, Kaplan V. Prevalence of chronic medical conditions in Switzerland: exploring estimates validity by comparing complementary data sources. BMC Public Health. 2014;14:1157.

25. Factors influencing health: Body weight by Body mass index (BMI) [http:// www.bfs.admin.ch/bfs/portal/en/index/themen/14/02/02/key/02.html]

26. Chiolero A, Peytremann-Bridevaux I, Paccaud F. Associations between obesity and health conditions may be overestimated if self-reported body mass index is used. Obes Rev. 2007:8:373-4

27. Faeh D, Marques-Vidal P, Chiolero A, Bopp M. Obesity in Switzerland: do estimates depend on how body mass index has been assessed? Swiss Med Wkly. 2008:138:204-10.

28. Aeberli I, Henschen I, Molinari L, Zimmermann MB. Stabilisation of the prevalence of childhood obesity in Switzerland. Swiss Med Wkly. 2010;140: w13046. 
29. Panczak R, Zwahlen M, Woitek U, Rühli FJ, Staub K. Socioeconomic, temporal and regional variation in body mass index among 188,537 Swiss conscripts born between 1986 and 1992. PLoS One. 2014;9:e96721.

30. Olds T, Maher C, Zumin S, Peneau S, Lioret S, Castetbon K, et al. Evidence that the prevalence of childhood overweight is plateauing: data from nine countries. Int J Pediatr Obes. 2011;6:342-60.

31. Stronski-Huwiler S, Stamm HP, Frey D, Christen L, Christen S, Takken-Sahli K LM: Übergewicht und Adipositas bei Kindern und Jugendlichen im Kanton Zürich. Gesundheit, Gesundheitsförderung und Gesundheitswes im Kant Zürich;Institut für Sozial- und Präventivmedizin der Universität Zürich 2013(Mai):9-16.

32. Murer SB, Saarsalu S, Zimmermann MB, Aeberli I. Pediatric adiposity stabilized in Switzerland between 1999 and 2012. Eur J Nutr. 2013;53(3): 865-75

33. Stamm H, Gebert A, Guggenbühl L, Lamprecht M. Excess weight among children and adolescents in Switzerland-prevalence and correlates for the early 2010s. Swiss Med Wkly. 2014;144:w13956.

34. Chappuis A, Bochud M, Glatz N, Vuistiner P, Paccaud F, Burnier M. Swiss survey on salt intake: main results. Lausanne: Federal Office of Public Health; 2011.

35. Ogna A, Forni Ogna V, Bochud M, Paccaud F, Gabutti L, Burnier M. Prevalence of obesity and overweight and associated nutritional factors in a population-based Swiss sample: an opportunity to analyze the impact of three different European cultural roots. Eur J Nutr. 2014;53:1281-90.

36. Ledergerber M, Steffen T. Prevalence of overweight and obesity in children and adolescents from 1977 to 2009 - examination of the school medical data of more than 94,000 school-age children in the city of Basel (Switzerland). Gesundheitswesen. 2011;73:46-53.

37. Guessous I, Joost S, Jeannot E, Theler J-M, Mahler P, Gaspoz J-M. A comparison of the spatial dependence of body mass index among adults and children in a Swiss general population. Nutr Diabetes. 2014;4:e111

38. Kopelman PG. Obesity as a medical problem. Nature. 2000;404:635-43.

39. Campion J, Milagro Fl, Martinez JA. Individuality and epigenetics in obesity. Obes Rev. 2009:10:383-92

40. Fletcher JM. The effects of smoking cessation on weight gain: new evidence using workplace smoking bans. Forum Heal Econ Policy. 2014;17:105-29.

41. Nesbit KC, Kolobe TH, Sisson SB, Ghement IR. A model of environmental correlates of adolescent obesity in the United States. J Adolesc Health. 2014; 55:394-401.

42. Drewnowski A, Moudon AV, Jiao J, Aggarwal A, Charreire H, Chaix B. Food environment and socioeconomic status influence obesity rates in Seattle and in Paris. Int J Obes. 2014;38:306-14.

43. Jilcott Pitts SB, Keyserling TC, Johnston LF, Smith TW, McGuirt JT, Evenson $K R$, et al. Associations between neighborhood-level factors related to a healthful lifestyle and dietary intake, physical activity, and support for obesity prevention polices among rural adults. J Community Health. 2014; 40(2):276-84.

44. O'Connor TM, Cerin E, Lee RE, Parker N, Chen T-A, Hughes SO, et al. Environmental and cultural correlates of physical activity parenting practices among Latino parents with preschool-aged children: Niños Activos. BMC Public Health. 2014;14:707.

45. Staub K, Woitek U, Rühli F. Impact and pitfalls of conscription data. In: Hermanussen M, editor. Auxology: studying human growth and development. Stuttgart: Schweizerbart'sche Verlagsbuchhandlung; 2013. p. 146-9.

46. Abel T, Hofmann K, Schori D. Social and regional variations in health status and health behaviours among Swiss young adults. Swiss Med Wkly. 2013; 143(December):w13901.

47. Staub K, Rühli FJ, Woitek U, Pfister C. BMI distribution/social stratification in Swiss conscripts from 1875 to present. Eur J Clin Nutr. 2010;64:335-40.

48. Neovius M, Rasmussen F. Place of residence and obesity in 1,578,694 young Swedish men between 1969 and 2005. Obesity. 2008;16:671-6.

49. Karnehed NEK, Rasmussen F, Hemmingsson T, Tynelius P. Obesity in young adulthood is related to social mobility among Swedish men. Obesity (Silver Spring). 2008;16:654-8.

50. Schober E, Rami B, Kirchengast S, Waldhor T, Sefranek R. Recent trend in overweight and obesity in male adolescents in Austria: a population-based study. Eur J Pediatr. 2007;166:709-14.

51. Rami B, Schober E, Kirchengast S, Waldhor T, Sefranek R. Prevalence of overweight and obesity in male adolescents in Austria between 1985 and 2000. A population based study. J Pediatr Endocrinol Metab. 2004;17:67-72.
52. Poglitsch M, Kefurt R, Mittlböck M, Bohdjalian a, Langer FX, Ludvik B, et al. Prevalence of obesity and overweight in male 18-year-olds in Austria from 2006 to 2010: an update. Eur Surg. 2011;43:181-6.

53. Toschke AM, Ludde R, Eisele R, von Kries R. The obesity epidemic in young men is not confined to low social classes-a time series of 18-year-old German men at medical examination for military service with different educational attainment. Int J Obes. 2005;29:875-7.

54. Engeland A, Bjorge T, Selmer RM, Tverdal A. Height and body mass index in relation to total mortality. Epidemiology. 2003;14:293-9.

55. Panczak R, Woitek U, Rühli F, Staub K. Regionale und sozio-ökonomische Unterschiede im Body Mass Index (BMI) von Schweizer Stellungspflichtigen 2004-2012. Zürich: Projektschlussbericht zuhanden des Bundesamtes für Gesundheit (BAG); 2013.

56. Militärärztlicher Dienst [http://www.lba.admin.ch]

57. BMl classification [http://apps.who.int/bmi/index.jsp?introPage=intro_3.html]

58. National Institutes of Health. Clinical guidelines on the identification, evaluation, and treatment of overweight and obesity in adults: executive summary. Expert Panel on the Identification, Evaluation, and Treatment of Overweight in Adults. Am J Clin Nutr. 1998;68:899-917.

59. International Standard Classification of Occupations (ISCO) [http://www.ilo. org/public/english/bureau/stat/isco/]

60. Hallström L, Vereecken C a, Ruiz JR, Patterson E, Gilbert CC, Catasta G, et al. Breakfast habits and factors influencing food choices at breakfast in relation to socio-demographic and family factors among European adolescents. The HELENA Study. Appetite. 2011;56:649-57.

61. Panczak R, Galobardes B, Voorpostel M, Spoerri A, Zwahlen M, Egger M. A Swiss neighbourhood index of socioeconomic position: development and association with mortality. J Epidemiol Community Health. 2012;66:1129-36.

62. Schrödle B, Held L. A primer on disease mapping and ecological regression using INLA. Comput Stat. 2010;26:241-58.

63. Blangiardo M, Cameletti M, Baio G, Rue H. Spatial and spatio-temporal models with R-INLA. Spat Spatiotemporal Epidemiol. 2013;7:39-55.

64. Gómez-Rubio V, López-Quílez A. Statistical methods for the geographical analysis of rare diseases. In: de la Paz Posada M, Groft SC, editors. Rare diseases epidemiology, vol. 686. Dordrecht: Springer Netherlands; 2010. p. 151-71. Advances in Experimental Medicine and Biology.

65. Besag J, York J, Mollié A. Bayesian image restoration, with two applications in spatial statistics. Ann Inst Stat Math. 1991;43:1-20.

66. Earnest A, Morgan G, Mengersen K, Ryan L, Summerhayes R, Beard J. Evaluating the effect of neighbourhood weight matrices on smoothing properties of Conditional Autoregressive (CAR) models. Int J Health Geogr. 2007:6:54.

67. Bernardinelli L, Clayton D, Montomoli C. Bayesian estimates of disease maps: how important are priors? Stat Med. 1995;14:2411-31.

68. Assareh H, Ou L, Chen J, Hillman K, Flabouris A, Hollis SJ. Geographic variation of failure-to-rescue in public acute hospitals in New South Wales, Australia. PLoS One. 2014;9:e109807.

69. Rue H, Martino S, Chopin N. Approximate Bayesian inference for latent Gaussian models by using integrated nested Laplace approximations. J R Stat Soc Ser B (Stat Methodol. 2009;71:319-92.

70. Spiegelhalter DJ, Best NG, Carlin BP, van der Linde A. Bayesian measures of model complexity and fit. J R Stat Soc Ser B (Stat Methodol. 2002;64:583-639.

71. Bivand RS, Pebesma EJ, Gómez-Rubio V. Applied spatial data analysis with R, vol. 1. New York Heidelberg Dordrecht London: Springer; 2013.

72. The R Core Team: R. A Language and Environment for Statistical Computing. Vienna, Austria; 2014. ISBN 3-900051-07-0. http://www.R-project.org.

73. Brewer CA, Campbell AJ. Beyond Graduated Circles: Varied Point Symbols for Representing Quantitative Data on Maps. Cartogr Perspect 1998;29:6-25.

74. Harrower M, Brewer CA. ColorBrewer.org: An online tool for selecting colour schemes for maps. Cartogr J. 2003:40:27-37.

75. Regional portraits: Cantons [http://www.bfs.admin.ch/bfs/portal/en/index/ regionen/kantone.html]

76. Henneberg M, Veitch D. Is obesity as measured by body mass index and waist circumference in adult australian women 2002 just a result of the lifestyle? Hum Ecol. 2005;13:85-89.

77. Burkhauser RV, Cawley J. Beyond BMI: the value of more accurate measures of fatness and obesity in social science research. J Heal Econ. 2008;27:519-29.

78. Schneider HJ, Friedrich N, Klotsche J, Pieper L, Nauck M, John U, et al. The predictive value of different measures of obesity for incident cardiovascular events and mortality. J Clin Endocrinol Metab. 2010;95:1777-85. 
79. Keys A, Fidanza F, Karvonen MJ, Kimura N, Taylor HL. Indices of relative weight and obesity. J Chronic Dis. 1972;25:329-43.

80. Sebo P, Beer-Borst S, Haller DM, Bovier PA. Reliability of doctors' anthropometric measurements to detect obesity. Prev Med. 2008:47:389-93.

81. Flegal KM, Kit BK, Graubard BI. Body mass index categories in observational studies of weight and risk of death. Am J Epidemiol. 2014;180:288-96.

82. Fuchs VR. Reflections on the socio-economic correlates of health. J Health Econ. 2004;23:653-61.

83. Nomenklaturen - Medstat-Regionen, Klassifikation [http://www.bfs.admin.ch/ bfs/portal/de/index/infothek/nomenklaturen/blank/blank/medstat/02.html]

84. Staub K, Woitek U, Rühli FJ. Grenzüberschreitende Zusammenarbeit mit anthropometrischen und medizinischen Daten der Rekrutierung. Swiss Rev Mil Disaster Med. 2013;1:41-5.

85. Schrödle B, Held L. Spatio-temporal disease mapping using INLA. Environmetrics. 2011;22:725-34.

86. Moser A, Panczak R, Zwahlen M, Clough-Gorr KM, Spoerri A, Stuck AE, et al. What does your neighbourhood say about you? A study of life expectancy in 1.3 million Swiss neighbourhoods. J Epidemiol Community Heal. 2014; 68(12):1125-32.

Submit your next manuscript to BioMed Central and we will help you at every step:

- We accept pre-submission inquiries

- Our selector tool helps you to find the most relevant journal

- We provide round the clock customer support

- Convenient online submission

- Thorough peer review

- Inclusion in PubMed and all major indexing services

- Maximum visibility for your research

Submit your manuscript at www.biomedcentral.com/submit
Biomed Central 